\title{
Similarity Theory of Energy Conversion Processes
}

\author{
Etkin Valeri Abramovich \\ Research Center of Togliatti State University, Togliatti, Russian Federation
}

Email address:

etkin.v@mail.ru

\section{To cite this article:}

Etkin Valeri Abramovich. Similarity Theory of Energy Conversion Processes. International Journal of Energy and Power Engineering. Vol. 8, No. 1, 2019, pp. 4-11. doi: 10.11648/j.ijepe.20190801.12

Received: March 19, 2019; Accepted: April 23, 2019; Published: May 23, 2019

\begin{abstract}
On the basis of energodynamics as a unified theory of nonequilibrium processes of energy transfer and transformation, a theory of similarity of such processes is proposed. It includes dimensionless criteria of efficiency, constructive perfection and load of power plants and the criterial equations connecting these indicators. On its basis, universal load characteristics of thermal and non-thermal, cyclic and non-cyclic, direct and reverse machines are built, reflecting their efficiency with the load and losses from irreversibility. The examples confirming their justice are given.
\end{abstract}

Keywords: Thermal and Non-Thermal Machines, Energy Conversion Processes, Relative and Absolute Efficiency, Similarity Criteria, Criterial Equations, Universal Load Characteristics

\section{Introduction}

It is considered that the maximum efficiency of any reversible non-thermal machine is equal to unity, while for a heat engine it is limited by the thermal efficiency of the ideal Carnot cycle [1]. Such "discrimination" of heat engines is based on the persistent conviction that "heat and work are unequal in principle because work can directly increase any form of energy, while heat can only increase internal energy." To substantiate this provision, they usually refer to machines using mechanical or electrical energy, which, like nuclear energy, is referred to as "fully convertible (non-entropic)" [2].

Those who believe that "narrowing the idea of the impossibility of creating a second-order perpetual motion engine before asserting that the properties of the heat source are exclusive from a fundamental point of view are not justified" disagree with this opinion [3]. As it turns out upon closer examination, such statements are the result of a number of methodological errors: deviations from the thermodynamic formulation of the problem, nondiscrimination of technical and non-technical (orderly and disordered) work, as well as absolute and relative efficiency, arbitrariness in choosing the origin of the potentials of non-thermal forms of energy and etc. [4]. This becomes more obvious after the generalization of the nonequilibrium thermodynamics of relaxation processes $[5$,
6] to the processes of useful (targeted) transformation of any form of energy [7]. The unity of the laws of transformation of thermal and nonthermal forms of energy, which was discovered at the same time, made it possible to propose a theory of their similarity, similar to that in heat and mass transfer theory [8]. Below is a justification of the basic provisions of this theory and sets out its immediate consequences.

\section{Towards a Unified Theory of Energy Conversion Processes}

There is a whole class of non-equilibrium systems that are difficult or even impossible to dismember into sources and receivers of heat, matter, charge, etc., as was done in the theory of heat engines. Such are, for example, complexly deformed bodies, homogeneous chemically reacting mixtures, polarized or magnetized media, dissociated and ionized gases, continuums with a different angular velocity of rotation, etc. In these cases, it becomes necessary to use the mathematical apparatus of non-equilibrium thermodynamics of the continuum. However, this turned out to be necessary not only to switch from thermostatics, which is essentially classical thermodynamics [1], to thermokinetics [9], which operates with energy flows and their material carriers, but also to generalize the existing theory of irreversible processes $[5,6]$ to systems, doing useful work 
[10]. Both of these tasks were solved in energodynamics [11] by building it not on the basis of the local equilibrium hypothesis and $d S / d t$ principle of entropy increase, but on a more general basis of the energy conservation law proposed by N. Umov in 1873 :

$$
d U / d t=-\int \nabla \cdot \mathrm{j}_{u} d V
$$

Where $\mathrm{j}_{u}$ is the energy flux density through the vector element $d$ f of the closed surface of the system with volume $V$ in the direction of the outward normal.

To express the energy flux density through the parameters of an inhomogeneous system as a whole, we use the combined equation of the 1 st and 2 nd principles of locally equilibrium thermodynamics in the form [12]:

$$
\mathrm{du}=\Sigma_{\mathrm{i}} \psi_{\mathrm{i}} \mathrm{d} \theta_{\mathrm{i}}
$$

Where $u$ is the specific internal energy of the system; $\psi_{i}-$ generalized potentials such as absolute temperature $T$, pressure $p$, chemical potential of the $k$ th component of the system $\mu_{k}$, and so on; $\theta_{i}$ are specific values of generalized coordinates $\Theta_{i}$ of entropy type $S$, volume $V$, number of moles of the $k$ th substance $N_{k}$, etc., hereinafter referred to as energy carriers for brevity. In this case, the flow of energy $\mathrm{j}_{u}$ can be represented in a compact form as the sum of its components:

$$
\mathrm{j}_{u}=\Sigma_{i} \psi_{i j} \mathrm{j}_{i}
$$

Where each of them is represented as a product of the generalized potential $\psi_{i}$ by the flux density $\mathrm{j}_{i}$ of the corresponding energy carrier. If we now decompose $\nabla \cdot \mathrm{j}_{u}=$ $\Sigma_{i} \nabla \cdot\left(\psi_{i} \mathrm{j}_{i}\right)$ into two components $\Sigma_{i} \psi_{i} \nabla \cdot \mathrm{j}_{i}+\Sigma_{i} \mathrm{j}_{i} \nabla \psi_{i}$, the energy conservation law (1) takes the form:

$$
d U / d t=-\Sigma_{i} \int \psi_{i} \nabla \cdot \mathrm{j}_{i} d V-\Sigma_{i} \int \mathrm{j}_{i} \cdot \nabla \psi_{i} d V
$$

By taking out the sign of the integral in (4) some average value $\Psi_{i}$ of the potential $\psi_{i}$ and its gradient $\mathrm{X}_{i}=-\nabla \Psi_{i}$, we finally get [11]:

$$
d U / d t \equiv \Sigma_{i} \Psi_{i} d \Theta_{i} / d t-\Sigma_{i} \mathrm{X}_{i} \cdot \mathrm{J}_{i},
$$

Where $d \Theta_{i} / d t \equiv \int \nabla \cdot \mathrm{j}_{i} d V$ и $\mathrm{J}_{i} \equiv \int \mathrm{j}_{i} d V$.

It is easy to see that in homogeneous systems $\left(\mathrm{X}_{i}=0, \Psi_{i}=\psi_{i}\right)$, identity (5) goes into the basic equilibrium thermodynamics equation $d U=\Sigma_{i} \psi_{i} d \Theta_{i}$, as expected. However, now the second sum of terms appears in it, characterizing the power (productivity) of $N$ real energy conversion processes from some $i$-th form $N_{i}=\mathrm{X}_{i} \cdot \mathrm{J}_{i}$ в $j$-ю to $j$-th $N_{j}=\mathrm{X}_{j} \cdot \mathrm{J}_{j}$. At the same time, the thermodynamic forces $\mathrm{X}_{i}$ and the fluxes $\mathrm{J}_{i}$, as generalized rates of energy transfer processes $\Theta_{i}$, are not based on the principle of increasing entropy $d S / d t=\Sigma_{i} X_{i} \cdot J_{i}>0$, but from a more general expression of the energy conservation law. Due to this, the theory of irreversible processes (TIP) does not exclude from consideration any (reversible or irreversible) part of real processes and extends to the vector processes of energy conversion.

It is important that in this case the thermodynamic forces $\mathrm{X}_{i}$-acquire a single meaning of the field strengths of the potentials $\Psi_{i}$, i.e. the specific forces $\mathrm{F}$, referred to the unit of energy carrier $\Theta_{i}$, and the flows $\mathrm{J}_{i}=\Theta_{i} \mathrm{v}_{i}$ are the single meaning of their momentum, where $v_{i}$ is the average speed of their transfer. This greatly facilitates the understanding of the essence of the processes.

\section{The Unity of the Processes of Energy Transfer and Transformation}

Introduction of time $t$ and power (productivity) of the process $N$ as physical parameters into the equations of thermodynamics allowed using its mathematical apparatus to establish the general laws of non-static (occurring at a finite velocity) energy conversion processes [11]. Consider for this purpose an arbitrary system of volume $V$, carrying out the steady process of converting some $i$-th form of energy into $j$-th. This process is described by the $\Sigma_{i} \int_{i} \cdot \nabla \psi_{i} d V$ terms of equation (4).

We represent the volume element $d V$ as the product of the vector element of its section $d$ f, normal to the flow of the primary energy carrier $\mathrm{J}_{i}$, and the element $d \mathrm{n}$ of the normal to it $(d V=d \mathrm{f} \cdot d \mathrm{n})$. Considering that in a steady stream $\left((d \mathrm{n} \cdot \nabla) \psi_{\mathrm{i}}\right.$ $=d \psi_{i}$, the expression $\int \mathrm{j}_{i} \cdot \nabla \psi_{i} d V$ can be given the form

$$
N_{i}=\left(\int \mathrm{j}_{i} \cdot d \mathrm{f}\right) d \psi_{\mathrm{i}}=-\int J_{i} d \psi_{i}=J_{i}\left(\psi_{i}{ }^{\prime}-\psi_{i}\right)^{\prime},
$$

Where $J_{i}$ is the "transit" flow of energy that crosses the system without changing its value; $\psi_{i}{ }^{\prime}, \psi_{i}{ }^{\prime \prime}$ - the potentials of the energy source at the input and output of the system.

This situation can be illustrated by the example of an electric machine, in which the input current, as is well known, is equal to the output current. This is just as obvious for a cyclic heat engine since in a circular process the increase in entropy in the process of heat supply $\Delta S$ is equal to its loss during heat removal to the heat sink. Therefore, in the steady state, the entropy fluxes $J_{s}=\Delta S / \Delta t$ at the input and output of the installation are also the same.

Thus, a necessary condition for the implementation of the transformation process of any $i$-th form of energy is the presence in the energy-converting system of a corresponding flow of energy carrier $J_{i}$ intersecting regions with different values of the generalized potential $\Psi_{i}$. For heat engines, this role is performed by the entropy flow $J_{s}$ from the heat source with temperature $T^{\prime}$ to the heat sink with temperature $T^{\prime \prime}$; for electric engine - electric current $J_{e}$, coupled with a drop in electric potential $\Delta \varphi$; for concentration elements, the flux $J_{k}$ intersects the boundaries of the system with different chemical potential $\mu_{k}$; for hydraulic machines, the flow of fluid $J_{m}$ between areas with different enthalpy values $h$, etc.

This distinctive feature of energy transformation processes - transfer of the corresponding thermostatic quantity $\Theta_{i}$ in the field of forces $\mathrm{X}_{i}$ - is also characteristic of microinhomogeneous systems. Indeed, the very notion of the flow of a quantity across the system's borders suggests that there are areas in it, from where the flow "flows out" and where it "flows in", that is, subsystems that change their properties in the opposite way during energy conversion. The role of such subsystems can be played by electrons and "holes" in 
semiconductors, positive and negative ions in electrolytes, opposite charges or poles of electric and magnetic dipoles, elements of a volume of elastically deformable bodies moving in opposite directions, etc.

This means that any system involved in the process of energy conversion (be it a source of technically suitable energy, a transforming device or an object of work) should always be considered as a heterogeneous system, the various parts of which change their state in the opposite way. Such changes of state representeded in the widest sense that "compensation" for the process of energy conversion, the need of which was proved by R. Clausius [13].

This position in accordance with (6) is valid for both the $i-$ th (convertible) and the $j-$ th (converted) form of energy. This means that any energy conversion device (both cyclic and noncyclic) is a system "penetrated" by the energy carrier flows of both the converted $J_{i}$ and the converted $J_{j}$ forms of energy. Such a "two-line" system in electrical engineering is depicted as a "quadrupole", i.e. a a device with two pairs of input and output terminals. For clarity, Figure 1 shows the electrical "equivalent circuit" of such an energy conversion system.

The energy conversion device depicted in this Figure (the central part of the Figure) seems to be "penetrated" by two dissimilar energy carrier flows $J_{i}$ and $J_{j}$. Thereby it is emphasized that this device interacts with two objects: a source of technically suitable energy (exergy) and with the object of work. The principal difference of the quadrupole from the concept of a heat engine adopted in thermostat as a combination of heat source, working fluid and heat sink is that in the quadrupole the transforming device (analog of the working fluid) is in contact with two "expanded" systems, each of which is non-equilibrium and sources and receivers of the energy carrier (like an extended system).

The transforming device (analog of the working fluid) is in contact with two "expanded" systems, each of which is nonequilibrium and sources and receivers of the energy carrier (like an extended system).

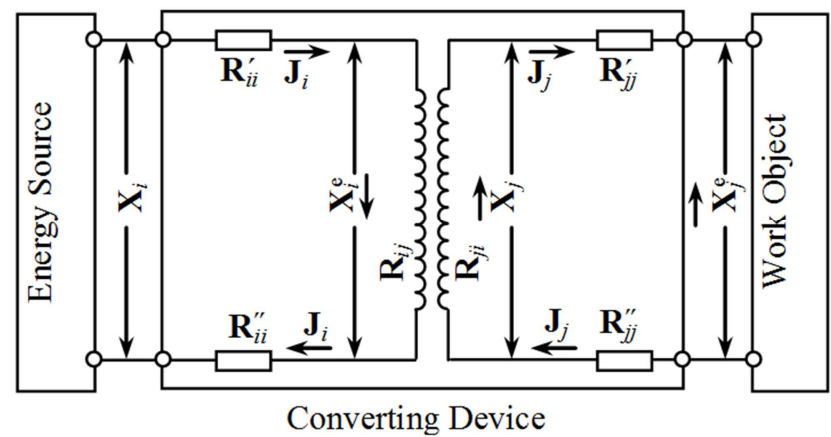

Figure 1. Electrical Equivalent Circuit to energy Converting Device.

The task of the energy conversion device in such a scheme is to use spatial heterogeneity in some material objects (energy source) to create heterogeneity in others (object of work).

The functional division of the material objects involved in the energy conversion process emphasizes the indissoluble unity of the energy transfer and conversion processes and provides the necessary generality of the problem of the thermodynamic perfection of the energy conversion process in thermal and non-thermal, cyclic and non-cyclic machines.

\section{Criteria for the Efficiency of Energy Conversion Devices}

The efficiency of the energy conversion device can be evaluated in various ways. For heat engines, one of them, called thermal efficiency $\eta_{t}$, was proposed by S. Karno [14] and was expressed by the ratio of the work of the machine $W$ to the amount of heat supplied from the source $Q_{1}$. Later, it began to be expressed as the ratio of the power of the machine $\mathrm{N}$ to the heat flow $J_{q}$ supplied from a hot source and expressed in terms of "average thermodynamic" (medium-integral) temperatures of heat supply $\bar{T}_{1}$ and removal $\bar{T}_{2}:$ и: $\eta_{t}=1-\bar{T}_{2}$ $/ \bar{T}_{1}$ [15]. This indicator belongs to the class of so-called "absolute efficiency". It admits a generalization and nonthermal machines if the power of the converter of the i-th form of Ni energy is attributed to the energy flow at its input $J_{i}$ ) [16]:

$$
\eta_{i}=N_{i} / J_{i} \bar{\psi}_{\mathrm{i}}^{\prime}=1-\bar{\psi}_{\mathrm{i}}^{\prime \prime} / \bar{\psi}_{\mathrm{i}}^{\prime}
$$

Where $\bar{\psi}_{\mathrm{i}}^{\prime}$ and $\bar{\psi}_{\mathrm{i}}^{\prime \prime}$ - mean integral values of the generalized potential at the input and output of the installation.

The magnitude of the absolute efficiency does not depend on the type of energy being converted, nor on the design of the machine, nor on the nature of its working body. Therefore, it is not applied to non-thermal machines, especially since the origin of the parameters is arbitrary (unlike the absolute temperature $T$ ) and when $\bar{\psi}_{\mathrm{i}}^{\prime \prime}=0$, it can reach $100 \%$. The so-called "relative" efficiency $\eta_{o i}$ has a different meaning. In classical thermodynamics, this concept is introduced to assess the perfection of only certain processes, for example, the processes of expansion of the working fluid. Such efficiencies have the meaning of the relation actually made in the process of $W_{i}$ to its theoretically possible value of $W_{i}^{t}$ [15]. All other disciplines, with the exception of thermodynamics, operate with such (relative) efficiency. For machines performing one kind of useful work, these efficiency values really only reflect the degree of perfection of the installation itself. This circumstance more than once served as a source of misunderstanding.

Herewith, the speaker does not even think that the socalled "waste with heat loss" can only be attributed to the heat $Q_{2}-Q_{2}{ }^{\text {min }}$ given over the theoretical minimum $Q_{2}{ }^{\text {min }}$ as a necessary condition for the energy conversion process itself.

To eliminate this misunderstanding and methodologically unified approach to converters of any form of energy, the concept of relative efficiency should be generalized to all types of energy-converting devices. As such a universal measure, energy dynamics proposes to consider in accordance with (5) the power ratio at the output $N_{j}$ and the input of the $N_{i}$ converting device [11]:

$$
\eta_{N}=N_{j} / N_{i}=-\mathrm{X}_{j} \cdot \mathrm{J}_{j} / \mathrm{X}_{i} \cdot \mathrm{J}_{i} \cdot\left(0<-\mathrm{X}_{j} \cdot \mathrm{J}_{j}<\mathrm{X}_{i} \cdot \mathrm{J}_{i}\right)
$$

We will call the relation (8) "power" efficiency. It 
differs from the exergy efficiency, expressed by the ratio of exergy flows (technically suitable energy) at the output and installation of the installation [15], by the absence of "transit" (not participating in energy conversion) components. That is why $\eta_{N}$ reflects the true thermodynamic perfection of the energy-converting device as the degree of its realization of the possibilities that the source of technically suitable (free) energy gives it, taking into account the irreversibility of energy transfer processes outside and inside the installation.

A quick glance at expression (8) is enough to understand that it, unlike absolute efficiency, vanishes twice: at $\mathrm{J}_{j}=0$ and at $\mathrm{X}_{j}=0$. This means that power efficiency takes into account the mode of operation of the installation. Another advantage of $\eta_{N}$ lies in its universality, that is, its applicability to the analysis of thermal and non-thermal, cyclic and non-cyclic, singlepurpose and multi-purpose, direct and inverse machines. Further, this efficiency takes into account all types of losses, both during energy transfer from a source to a conversion device, and during energy conversion in this device itself. Finally, this efficiency is the only possible indicator of the perfection of the installation in cases where it is impossible to separate the sources and receivers of the converted form of energy. installation.

\section{Phenomenological Laws of Energy Conversion Processes}

The most important difference in the energy dynamic approach to the study of energy conversion processes is to take into account the relationship of heterogeneous flows $\mathrm{J}_{i}$. As in the TNP, energy dynamics takes into account that each of these flows depends not only on the same force $\mathrm{X}_{i}$ or $\mathrm{X}_{j}$, as in the laws of Fourier, Ohm, Fick, etc., but also on the "alien" force $\mathrm{X}_{j}$ energy. In the TIP, this is reflected in the socalled "phenomenological" (based on experience) Onsager laws of the form [6]:

$$
\mathrm{J}_{i}=\Sigma_{i} R_{i j} \mathrm{X}_{j}
$$

Where $R_{i j}$ - phenomenological coefficients of resistance to the $i$-th flow from the side of the same $\mathrm{X}_{i}$ or "alien" $\mathrm{X}_{j}$ forces.

It is easy to see that when finding the flows $\mathrm{J}_{i}$ and the forces $\mathrm{X}_{i}$ on the basis of the production of entropy $d S / d t$, all the terms (9) are positive. This is fully applicable to relaxation processes considered in the "quasithermodynamics" by L. Onzager. However, they do not correspond to energy conversion processes, for which, according to (8), the sign of the terms $\mathrm{X}_{i} \cdot \mathrm{J}_{i}$ and $\mathrm{X}_{j} \cdot \mathrm{J} j$ is opposite. Therefore, the kinetic equations of the energy conversion process have the form different from (9):

$$
\begin{aligned}
& J_{i}=L_{i j} X_{i}-L_{i j} X_{j} . \\
& J_{j}=L_{j i} X_{i}-L_{j j} X_{j} .
\end{aligned}
$$

This nature of the kinetic equations is best illustrated by the example of a transformer, the circuit of which corresponds to Figure 1. In it, as is known, as the overcome forces $X_{j}$ increase (with approaching the "idle" mode), the current $J_{i}$ decreases in both the primary and secondary $J_{j}$ circuits. This interconnection of If the coefficients $R_{i i}, R_{i j}, R_{j i}$ and $R_{j j}$ do not depend on the flows associated with them (that is, laws (10) and (11) are linear), they can be represented in a dimensionless form that does not contain any phenomenological coefficients at all. To do this, we consider two limiting cases: the engine idling mode, in which $J_{j}=0$, and the force $X_{j}$ reaches its limit value $X_{j o}$ (idling voltage), and the "short circuit" mode, when $X_{j}=0$ and $J_{j}$ is maximum (equal to the current short closure $J_{j k}$ ) Expressing $X_{j}$ and $J_{j k}$ in accordance with equations (10) and (11) in terms of phenomenological coefficients, we find in conditions of the the constancy of $X_{i}[11]$ :

$$
X_{j} / X_{j o}+J_{j} / J_{j k}=1
$$

Such a "dimensionless" form of phenomenological laws further emphasizes the unity of the processes of transformation of any form of energy.

\section{Criteria for the Similarity of Energy Converters}

The independence of the laws of energy conversion (12) from the instrumentation design of installations carrying out such processes makes it possible to lay the foundations of the theory of similarity of linear energy conversion systems. The mathematical model of such a system includes, along with equations (4) and (5), the conditions for the uniqueness of the processes under study. The latter containss [11]:

a) resistance coefficients $R_{i j}(i, j=1,2)$, which characterize the transport properties of the system (analogue of thermophysical properties in the theory of heat and mass transfer);

b) the boundary conditions determined in the case under consideration by the magnitude of the driving forces in the current mode $X_{i}, X_{j}$ or the flux values in these modes $J_{i}, J_{j}$;

c) the initial conditions specified by the magnitude of these forces or flows at the "idle" setting (at $J_{j}=0$ ) $X_{j o}$, $J_{i o}, J_{j o}$ and in the "short circuit" mode (at $\left.X_{j}=0\right) J_{i k}, J_{j k}$.

These conditions of uniqueness allow us to form a series of dimensionless criteria for the similarity of energy-transforming systems. We consider these equations together with the conditions of uniqueness. Putting $J_{j o}=0$, $X_{i}=X_{i o}, X_{i}=X_{i o}$, we find from them $X_{i o} / X_{i o}=R_{j j} / R_{i i} ; \mathrm{Jj}=$ $\mathrm{Xj} 0 / \mathrm{Rji} ; \mathrm{Jj}=(\mathrm{xj}-\mathrm{xj} 0) / \mathrm{rjj}$. Similarly, in the "short circuit" mode $\left(X_{j}=0, J_{j}=J_{j k}, J_{j}=J_{j k}\right)$ from (4) we have:: $J_{j k} / J_{i k}=R_{j i} / R_{j j}$. After substituting equations (3) and (4) into the expression of power efficiency (8) and a number of transformations, we obtain the expression:

$$
\begin{gathered}
\eta_{N}=\left(X_{j} / X_{j 0}\right) /\left[1+R_{i i} R_{j j} / R_{i j} R_{j i}\left(1-X_{j} / X_{j 0}\right)\right] \\
\Phi=R_{i j} R_{j i} / R_{i i} R_{j j .}
\end{gathered}
$$


This complex is similar to the ratio of reactive and active resistances, known in radio engineering as the quality factor of the circuit, and coincides (up to a temperature factor) with the "quality factor" $\Phi$, introduced by A. Ioffe as a generalizing characteristic of thermoelectric generators (TEG) [16]. Its value ranges from zero to infinity $(0<\Phi<\infty)$, increasing with decreasing "active" resistances (from the side of the scattering forces) $R_{i i}$ and $R_{j j}$ and increasing "reactive" resistances to $R_{j i}$ (from the side of "alien" forces). Like thermal resistances in heat transfer theory, these resistances depend on the transport properties of the system (sections and lengths of heat transfer lines, matter, charge, etc., on the properties of the materials used and the values of transfer coefficients, etc.), i.e. ultimately, from the constructive perfection of the installation, which depends indirectly on the costs in the process of its manufacture. The latter means that this criterion reflects the constructive perfection of the installation [7].

Another dimensionless criterion can be composed of the boundary conditions given by the value of the forces $X_{j}$, $X_{j o}$ or the flows $J_{j}, J_{j k}$ :

$$
B=J_{j} / J_{j k}=1-X_{j} / X_{j o}
$$

This criterion depends solely on the load of the installation (analogue of the "load current") and varies from zero in idle mode $\left(J_{j}=0\right)$ to unity in the "short circuit" mode $\left(X_{j}=0\right)$. Therefore, he was called by us the criterion of the load installation [7].

Using these criteria, expression (13) can be given the form of a criterion equation for the process of energy conversion:

$$
\eta_{N}=(1-B) /(1+1 / B \Phi)
$$

This equation establishes the dependence of the thermodynamic criterion of the the perfection of the installation on its load B with constant parameters of the source of technically acceptable energy. According to him, the relative efficiency of any linear energy converter under similar conditions (B, $\Phi=\mathrm{idem})$ is the same. For the convenience of reference, it is reasonable to call this provision the principle of similarity of power plants.

\section{Universal Load Characteristics of Energy Converters}

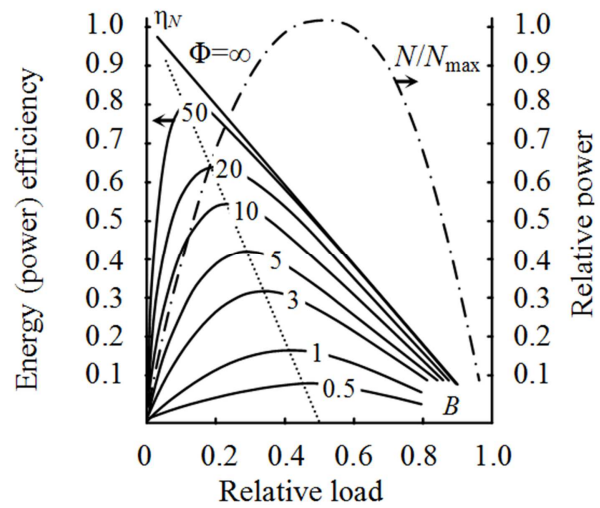

Figure 2. Universal load characteristics of linear energy converters systems.
As in hydrodynamic, the criterial equation of energy conversion processes (10) allows you to transfer the results of studies of the effectiveness of some technical systems to others (little studied). For convenience, it is advisable to build on the basis of the criterial equation (10) a generalized load characteristic of linear energy-transforming systems [11] This characteristic is shown in Figure 2. Solid lines show here the power efficiency of the machine $\eta_{\mathrm{N}}$ plotted against the load criterion $B$ for different values of the criterion $\Phi$, while dotted lines - the output power $N_{j}$ plotted against the load. As follows from the Figure, in the absence of energy losses $(\Phi=\infty)$ and with the the quasi-static character of its conversion $(B \rightarrow 0)$ the machine efficiency reaches, as should be expected, unity. However, in all other cases the power efficiency becomes zero twice: in "no-load" ( $B=0, J_{j}$ $=0)$ and "short circuit" $\left(B=1, X_{j}=0\right)$ operations. This ensues from taking into account, along with the irreversible power interchange and friction, also various "leaks" 1) of power occurring in also "no-load" operation of the machine, in particular, its auxiliary power. With the loads growing and the operation withdrawing from "no-load" conditions the efficiency rises and reaches a a maximum at a definite load. The efficiency maximums then lie on the same line connecting the operating conditions with "zero" output power $\left(B=0, \eta_{N}=1\right.$ и $\left.B=0,5, \eta_{N}=0\right)$. The efficiency maximums appeared are explained by varying relationship between the rates of useful and dissipative energy conversions in the system and show that all types of energy converters have the most economical loads usually adopted as nominal.

To find the nominal loads $B_{\mu}$, let us set the derivative of (10) with respect to $B$ equal to zero. Some transformations give:

$$
B_{\mathrm{H}}=(\sqrt{1+\Phi}-1) / \Phi
$$

According to this expression the power efficiency maximums lie on the same line connecting the points with the load $B=0.5$ and the efficiency $\eta_{N}=1$. The max efficiency value depends in this case on exclusively the $\Phi$-criterion value. In fact, substituting (17) into criterion equation (16) gives after simple transformations:

$$
\eta_{N}{ }^{\max }=(\sqrt{1+\Phi}-1) /(\sqrt{1+\Phi}+1)
$$

As can be seen, with the $\Phi$ - a factor of the machine rising (by additional investments as well), the nominal operating conditions shift toward decreasing loads. However, on partial loads (below nominal) the power efficiency drops in this case even more abruptly. In this respect, the deductions of thermokinetics also comply with a known provision that the operation of high-efficiency power machines on partial loads is inadvisable. To clarify how the load impacts on the output power of a machine, let us substitute (10) into the

1) This loss can not be taken into account by traditionally introducing the constant relative efficiencies of work-doing processes since this loss is equal to zero in "no-load" operation. 
relationship $N_{j}=N_{i} \eta_{N}$

$$
N_{j}=X_{j 0} J_{j k} B(1-B)
$$

Since at $B=0.5$ the power $N_{j}$ is maximal $\left(N_{j}=N_{j}^{\max }\right)$, the value $X_{j o} J_{j k}=4 N_{j}$, and instead of (19) one can write

$$
N_{j} / N_{j}^{\max }=4 B(1-B
$$

This plot is also shown on Figure 2 with a a dotted line. From this, it follows that at the efficiency $\eta_{\mathrm{N}}=1$ the output power of any heat engine is equal to zero due to the absence of heat exchange between the heat well and working medium and reaches a maximum at a relative load of $B=0.5$. The efficiency of the engine corresponding to these loads can be found by substituting this load value into (16):

$$
\eta_{(N=\max )}=1 /(2+4 / \Phi)
$$

According to this expressio, $\mathrm{n}$ the power efficiency of linear energy converters at max power conditions does not exceed $50 \%$ for all forms of energy. For all that the difference in efficiencies of machines with different $\Phi$-factors is leveling off with approaching the max-output power-operating conditions and may become practically indistinguishable. This fact reveals the lack of prospects in pursuing high efficiencies for power machines intended for peak-load or poweraugmentation operations. Figure 2 also shows that with the $\Phi$-factor of a machine rising, the dependence of its efficiency on load grows more perceptible, while the operating conditions of max efficiency and max output power are more and more drifting apart.

Another important conclusion from loading characteristics is a a possibility of a finding of a criterion of good quality (constructibility) of installation $F$ by maximum position moshchnostnogo efficiency $\eta_{N}$. Equating zero derivativess of expression (10), we will find:

$$
\Phi=\left(1-2 B_{\text {опт }}\right) / B_{\text {опт }}^{2}
$$

Where $B_{\text {опт }}$ - the optimum loading corresponding to a maximum of efficiency $\eta_{N}$.

The universal load characteristics are very convenient when comparing machines of different types for choosing the most challenging of them. Since such an assessment depends on a load of a machine ranging considerably in operation, the comparison needs the load characteristics to be known otherwise being insufficient. Let us show this by the the example of a hydrazine-oxygen electrochemical current generator (ECG). When operating this fuel cell, a no-load voltage (EMF) was obtained as $X_{j o}=1.6 \mathrm{~V}$ which decreases with load growth and becomes $X_{j}=0.85 \mathrm{~V}$ at a power of $N_{j}=2 \mathrm{~kW}$. Fuel utilization factor and oxidant utilization factor for this unit are $\eta_{\mathrm{f}}=0.8$ and $\eta_{\mathrm{ox}}=0.9$, respectively [19]. To find the ECG power efficiency, let us first calculate the fuel element relative load $B=1-X_{j} / X_{j o}=$ $1-0.85 / 1.6=0.47$. For ideal ECG (with the $\Phi=\infty$ ) under such a load according to Figure $15.2 \eta_{N}$ does nor exceed
0.51. Allowing for the mean factor of reagents utilization as $\eta_{\mathrm{r}}=0.85$ gives the effective efficiency for this generator $\eta_{\mathrm{e}}$ $=\eta_{\mathrm{N}} \eta_{\mathrm{r}}=0.51 \cdot 0.85=0.43$. Detailed calculations with an exergy flow balance analysis give for this ECG the exergy efficiency $\eta_{e x}=0.41$ [20], i.e. quite close to $\eta_{\mathrm{e}}$. At the same time, such a proximate analysis reveals the importance of considering the loads to machines when investigating how far they are challenging. Should in the example considered the ECG effectiveness be estimated thru the max power efficiency $\eta_{N} \cong 0.85$, the situation would be cardinally outweighed on its side.

\section{Examples of the Power Plants Load Characteristics Similarity}

It is of interest to confirm the similarity of the load characteristics of installations of different types. As one example, consider the propulsion system of rocket space engines (RCD) [21]. The power of their engines $N_{j}$ is defined as the scalar product of the thrust force $\mathrm{R}$ and the rocket speed $\mathrm{v}$ relative to the starting (orbital) absolute speed $\mathrm{v}_{0}$, and the power delivered to the engine $N_{i}=G\left(h_{c}{ }^{*}-h_{\kappa}{ }^{*}\right)$ is the product of the gas flow $G$ by the difference of the product braking enthalpy combustion at the entrance to the combustion chamber $h_{\kappa}{ }^{*}$ and at the exit of the nozzle $h_{c}{ }^{*}$ [22]. After a series of transformations, the RDC efficiency $\eta_{N}$ can be represented as a criterion dependence [22].

$$
\eta_{\mathrm{N}}=2 \mathrm{~B}(1-\mathrm{B}) /\left[\mathrm{B}^{2}\left(\mathrm{w} / \mathrm{v}_{\mathrm{o}}\right)^{2} / 2 \eta_{\mathrm{oi}}+\mathrm{v}_{\mathrm{o}} / \mathrm{w}\right]
$$

Where $B=\mathrm{v}_{\mathrm{o}} / \mathrm{v}$ is the load criterion; $\mathrm{w}$ is the relative speed of the jet; $\eta_{o i}$ is the relative internal efficiency of the RCD nozzle.

This dependence for different values of specific thrust $R_{s}=$ $|\mathrm{R}| / G$ is shown in Figure 3. According to the Figure, the efficiency $\eta_{\mathrm{N}}$ vanishes twice: at $B=0$ and $B=1$. The first case corresponds to flights with near-light speeds when the the further increment of the flight speed becomes impossible $\left(J_{j}=J_{j k}\right.$, as in the "short circuit" mode). This situation is of theoretical interest, for example, for space rockets with solar sails [21], whose thrust drops to zero as the speed of flight approaches the speed of light. The opposite case $(B=1)$ is characteristic of any rocket engines at the moment of their launch when the flight speed is equal to the starting one $(\mathrm{v}=$ $\mathrm{v}_{\mathrm{o}}$ ). With increasing flight speed, the efficiency curve passes through a maximum, whose position depends on the ratio $\mathrm{v}_{\mathrm{o}} / \mathrm{w}$ and on the degree of perfection of internal processes in the engine [22].

As another example, let us compare the individual characteristic of the thermal emission energy converter (TEC), built on the basis of the universal load characteristic of Figure 2, with the experimentally found dependence of the power and the effective efficiency of one of these converters on the voltage at its output $\Delta \varphi[18]$. 


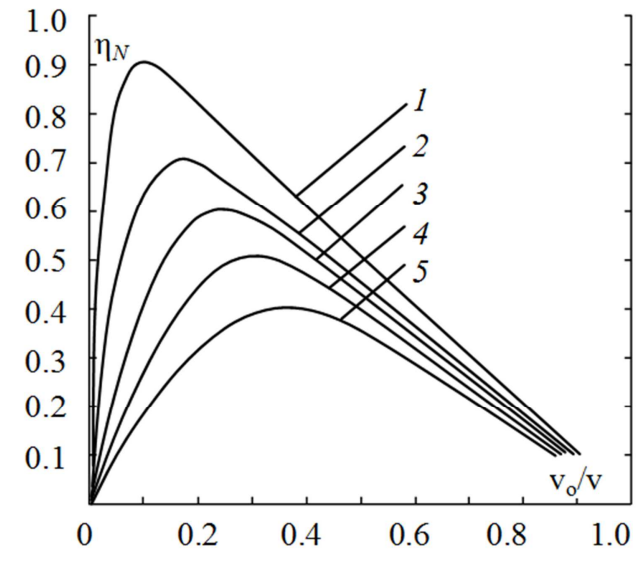

$1,2,3,4,5-\mathrm{R}_{\mathrm{s}} / \mathrm{v}_{0}=1.0 ; 0.5 ; 0.2 ; 0.15 ; 0.1$

Figure 3. Generalized Curves SBP Efficiency - Figureht Speed.

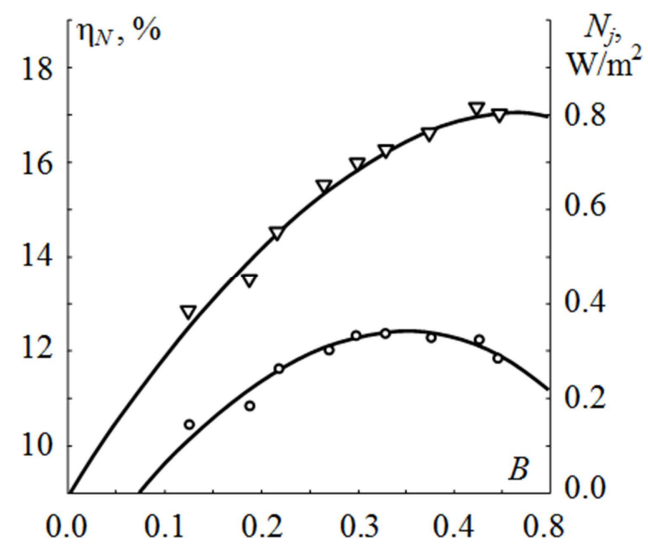

Figure 4. Load Characteristics of Thermionic energy Converters.

When finding the quality factor of the TEC, we will proceed from its characteristics, according to which the effective efficiency of TEC at Эe at cathode and anode temperatures of 2200 and $30^{\circ} \mathrm{C}$, respectively, is $11 \%$ maximum. Taking into account that at these temperatures the theoretical efficiency of the ideal TEC, $\eta_{\mathrm{t}}{ }^{\mathrm{K}} \approx 0,88$, we find that this effective efficiency corresponds to the power efficiency $\eta_{N}=12,5 \%$. This is enough for a series of curves in Figure 2 select and build an individual load characteristic of TEC. In order to compare with it the experimental dependence of the effective efficiency оте on the voltage at the output of the TEC, it is necessary to recalculate it into a generalized form $\eta_{\mathrm{N}}=\eta_{\mathrm{N}}(B)$. This is easy to do by relating the voltage at the TEC output $\Delta \varphi$ to the "no load" voltage (EMF TEC) $\Delta \varphi_{0}=0.9 \mathrm{~V}$, since for TEC, $B=1-\Delta \varphi / \Delta \varphi_{0}$. The results of such a recalculation are presented in Figure 4 . The light dots in this Figure indicate the experimental values of the effective efficiency, the dark ones indicate the specific power of TEC. As follows from the Figure, in general, the nature of the curves $\eta_{\mathrm{N}}=\eta_{\mathrm{N}}(B)$ and и $N_{j}=N_{j}(B)$ fully corresponds to the universal load characteristics. At the same time, it follows from Figure 4 that the tested TEG did not work optimally, which in this case corresponds to the loads $B$ $=0.3 \ldots 0.35$.
The fact of existence and differences in modes with maximum efficiency and maximum power is also confirmed by the well-known efficiency curves of electrical energy converters, characteristics of pumps and fans, internal combustion engines, etc.

Thus, the similarity theory takes another step towards approximating the results of thermodynamic analysis of various energy converters to reality. The next step should be the synthesis of the theory of similarity of power plants with thermoeconomics [24], aiming at measuring the savings caused by the increase in plant efficiency with the investment required for this. In Russia, this direction was developed thanks to the work of A. I. Andryushchenko [25] and his school [26].

\section{Conclusion}

1. Accounting for the kinetics of energy transfer and energy conversion processes from the standpoint of energodynamics as a further generalization of the theory of relaxation processes to the processes of useful transformation of any form of energy reveals the unity of the laws of transformation of thermal and non-thermal forms of energy. This refutes the traditional view of the exclusivity of heat engines and the universality of thermal efficiency as an indicator of their effectiveness.

2. From the standpoint of energodynamics, any energy conversion device appears as a two-flow system in which the flows and driving forces of the transformed and transformed forms of energy are interconnected by the Onsager-Casimir antisymmetric reciprocal relations. For such systems, thermal efficiency gives way to the exergy linking their powers.

3. The laws of energy transfer and transformation processes can be represented in a dimensionless form, which allows one to introduce a number of similarity criteria in the same way as in heat transfer theory. These criteria are the relative (power) efficiency of the energy converter, the criterion for the constructiveness of the power plants, depending on the coefficients such as electrical conductivity and thermal conductivity, and the load criterion, which depends on the mode of its operation.

4. The criterion equation of the energy conversion process allows us to construct a universal load characteristic of real energy-converting systems, independent of the form of the energy being converted, such as the energy-transforming installation (thermal and non-thermal, cyclic and non-cyclic, direct or inverse), as well as their hardware design.

5. The universal load characteristic detects the inversion of the relative power efficiency of any installation to zero twice: at idle and in the short circuit mode, the presence of modes with maximum efficiency and their discrepancy with the maximum power modes. This makes it easier to find such modes for existing plants and a comparative analysis of the efficiency of future power plants, taking into account their expected modes of operation. 


\section{References}

[1] Bazarov, I. P. Thermodynamics. NY, Pergamon Press, 1964.

[2] Exergic calculations of technical systems. / Etc. A. A. Dolinsky and V. M. Brodyansky). Kiev: Naukova Dumka, 1991. (In Russian)

[3] Gukhman A. A. On the foundations of thermodynamics. Izd.2-e. - Moscow, 1986.(In Russian).

[4] Etkin V. A. On the maximum efficiency of non-thermal engines // Heat Engineering. M.: Higher school., 3(1980). 4351.. (In Russian)

[5] De Groot S. R., Mazur R. Non Equilibrium Thermodynamics:- Amsterdam, 1962.

[6] Haase R. Thermodynamik der irreversiblen processe. Darmstadt, 1963.

[7] Etkin V. A. To the non-equilibrium thermodynamics of energy transformation systems. // Soviet. Journal of Appl. Physics, 6(1990).720-725 (translated from Izv. Sib. Otd. RAN-Tekhnika (Bulletin of Russian Acad. Science, Siberian BranchEngineering), 6(1990), pp. 120-125).

[8] Etkin V. A. To the similarity theory of power plants. //Atti del $49^{\circ}$ Congresso Nat. ATI.-Perugia, 4(1994). 433-443.

[9] Etkin V. Thermokinetics (Synthesis of Heat Engineering Theoretical Grounds).- Haifa, 2010. 334 p.

[10] Etkin V. A. Synthesis and new applications of the energy transfer and energy conversion theories. (Summary of thesis for doct. techn. sciences. //Moscow: State Techn. Univ. Press, 1998.- 35 pp. (in Russian).

[11] Etkin V. Energodynamics (Thermodynamic Fundamentals of Synergetics).- NY., 2011. 480 p.

[12] Etkin V. Principle of non-equilibrium processes counters directivity. // Reports by independent authors. 37(2016).86 94.

[13] Gyarmati I. Non-Equilibrium Thermodynamics. Field Theory and Variation Principles. - Springer -Verlag, 1970.

[14] Klausius R. Die mechanishe Wärmetheorie. Draundschweig, Bd. I, 1876

[15] Carnot $S$. Reflexions sur la puissance motrice du feu et sur les machines propres a developped cette puissance. Paris, 1824.

[16] Andryuschenko, A. I. Fundamentals of Technical Thermodynamics of Real Processes. 2 nd ed. M.: Higher. school, 1985. (In Russian)

[17] Etkin V. A. To the non-equilibrium thermodynamics of energy transformation systems. // Soviet. Journal of Appl. Physics, 1990.- 6, 720-725 (translated from Izv. Sib. Otd. RANTekhnika (Bulletin of Russian Acad. Science, Siberian BranchEngineering), 6(1990), 120-125).

[18] Onsager L. Reciprocal relations in irreversible processes // Phys. Rev. 237(14) 1931. P. 405-426; 238(12). 2265-2279.

[19] Tribus M. Thermostatics and thermodynamics. M.: Energy, 1970. (In Russian).

[20] Nesterov B. P., Korovin N. V., Brodyansky V. M. On Exergy Efficiency of Electrochemical Current Sources // Electrochemistry, 16(6), 1980. 814-820.

[21] Etkin V. A. Method of studying linear and non-linear irreversible processes. //Russian Journal of Physical Chemistry, 1991, 65(3), pp. 339-343 (translated from Zhurnal Fizicheskoi Khimii, 1991, 65, pp. 642-651).

[22] Favorsky O. N, Fishgoit V. V., Yantovsky E. I. Fundamentals of the theory of space electric propulsion systems. - M.: Higher school, 1970. (In Russian).

[23] Etkin V. A. To optimize the specific impulse of rocket engines. // News of universities. Aviation technology. 1(1999). 76-78. (In Russian).

[24] Tribus M., Evans R. B., Crellen G. L. Thermoeconomics. /Principles of desalination. - NY, Acad. Press, 23(1966).77101.

[25] Andryushchenko A. I. Methods of system thermodynamic research in heat and power engineering: Proc. Benefit. Ed. 2nd. - Saratov, GTU, 1996. (In Russian).

[26] Etkin V. A. Fundamentals of the theory of performance of power plants. // Proceedings of the XIII Int. Conf. "Improvement of energy systems and thermal power complexes", Saratov, 2016, 22-31. 